\title{
REVIEW
}

\section{The Novel Coronavirus 2019-nCoV: The Effects of Social Distancing and Partial Health Confinement on the Psychological State of Parkinson's Disease Patients}

\author{
Abderrahmane Achbani a (iD, Sofiane Ait wahmane a (iD), Zakaria Ouhaz ${ }^{\text {c c }}$, Mohamed Elatiqi a ${ }^{\text {a }}$ Hasnaa Sine ${ }^{\text {d (D), }}$ \\ Youssef Bouchriti $^{\text {e (D), Ahmed Belmouden }}{ }^{\text {a }}$ (D), Mohamed Nejmeddine a \\ ${ }^{\text {a }}$ Laboratory of Cell Biology and Molecular Genetics, Department of Biology, Faculty of Sciences, University Ibn Zohr, Agadir, Morocco. \\ ${ }^{\mathrm{b}}$ Department of experimental Psychology, University of Oxford, Tinsley Building, Mansfield Road, OX1 3SR, Oxford, UK. \\ ${ }^{\mathrm{c}}$ Ben M'Sik Faculty of Sciences, Hassan II University, Casablanca, Morocco. \\ ${ }^{\mathrm{d}}$ Faculty of Medicine and Pharmacy, University Mohamed V, Rabat, Morocco. \\ ${ }^{\mathrm{e}}$ Faculty of Sciences, University Ibn Zohr, Agadir, Morocco.
}

\begin{abstract}
Background: The novel coronavirus 2019-nCoV caused by SARS-CoV-2, has caused global, large and major public health issues. During the pandemic, different public health strategies were implemented in order to reduce the spread of this virus. These strategies consisted on increasing the frequency of hand-washing, using personal protection equipment, social distancing and restricting movements cities along with sealing national borders. During this crisis, Health Systems undergo important pressure and remarkable efforts were implemented to provide an efficacious reaction to this emergency. However, the actual global policy of diverting the attention exclusively to the COVID-19 pandemic and overshadowing other clinical conditions may have substantial negative implications. There are particular concerns around the increased vulnerability of patients living with a chronic disease, and this also includes neurological conditions like Parkinson's disease (PD). Aims: This review discusses the different outcomes of social distancing on PD patients and summarizes the circuitry of social behaviour in the context of PD. We consider the possible psychological and behavioral outcomes of quarantine and social isolation as part of national policies to limit the spread of the virus. Conclusion: In total, this evidence suggests that the drastic COVID19 pandemic measures could result in affective, cognitive and psychological alterations in these patients. Given this we suggest that patients with PD should be accompanied by their caregivers and encouraged to interact virtually with other family members and friends using different communication technologies. We also recommend healthcare providers to adopt telemedicine for outpatients visits and display educational programs for the patients.
\end{abstract}

Keywords: COVID19; Parkinson disease; Social Isolation; Confinement; Psychological Outcomes.

Correspondence: Achbani Abderrahmane, Laboratory of Cell Biology and Molecular Genetics Department of Biology Faculty of Sciences University Ibn Zohr,Agadir, Morocco : . Email: abderrahmane.achbani@edu.uiz.ac.ma

Copyright $\odot 2020$ Achbani A et al. This is an open access article distributed under the Creative Commons Attribution 4.0 International, which permits unrestricted use, distribution, and reproduction in any medium, provided the original work is properly cited.

\section{INTRODUCTION}

Parkinson's disease (PD) is one of the most common pathologies in the elderly, affecting 1 to $2 \%$ of the population over 50 years of age; it is also the second most common neurodegenerative disease in the world, after Alzheimer's.[1] PD was mainly identified by three cardinal motor signs: tremor, akinesia and rigidity. In the last decades, research has described a new cluster of symptoms called Non-Motor Symptoms (NMS). They include sensory alterations, signs of dysautonomia , sleep and neuropsychiatric/mood disorders.[2] The most common psychiatric perturbations reported in most neurodegenerative diseases are depression and anxiety, which can both occur early before the onset of motor symptoms.[3]

In December 2019, a new coronavirus (SARS-CoV-2) appeared, causing an outbreak of acute respiratory syndrome (COVID-19) in humans.[4] SARS-CoV-2 has rapidly spread across the globe; as a result, the World 
Health Organization (WHO) declared the associated disease a pandemic on March $11^{\text {th }}, 2020$. Until the completion of writing this paper, the COVID-19 is affecting 213 countries and territories. SARS-CoV-2 has infected 4,654,991 people and has caused 309,133 deaths.[5] In the absence of treatment, governments in collaboration with WHO have established strategies to limit the spread of the virus and protect Human life, one of the core measures that were implemented were social distancing and health containment.

Meanwhile, applying these measure for longer periods could result in serious psychological impacts such as anxiety, insomnia, panic behavior, fear, stress and hopelessness.[6]

The COVID-19 pandemic represents a worldwide major public health emergency, not only due to its high potential to spread and lethality, but also because of its potential consequences for public health in the medium and long term. The psychological Impact of the pandemic on the Parkinson's patients is the focus of the present review. We discuss the hypothesis that SARS CoV-2 infection could exacerbate the affective and cognitive symptoms of the disease, then we analyse its possible impact on the disease progression.

We think that the outcomes of social distancing and cities lockdown would exacerbate both motor and non-motor symptoms via increasing stress levels, anxiety and depression and reducing the physical activity of PD patients. Interestingly, it has been described that cognitive stress reduces the efficacy of dopaminergic medications on Parkinson's tremor ${ }^{[7]}$ Physical activity (PA) is widely advised as an adjunct intervention to improve PD symptoms and treatment efficiency.[8] Hence, Being stuck at home because of the confinement, may increase the severity of both the motor and non-motor PD symptoms. The purpose of this study, also, was to describe the neuronal pathways underlying social behavior in the context of PD.

\section{NEUROLOGICAL MANIFESTATIONS OF COVID19}

From previous studies on SARS-CoV, it has been shown that this virus infected the brain, including the brainstem, in both patients and experimental animals.[9] Following intranasal virus inoculation in mice, SARS-CoV or MERS-CoV entered the CNS, possibly through the olfactory nerves, and, importantly, the viruses were detected in the brain, but not in the lung, suggesting direct transfer to the CNS by olfactory nerves.[10] However, detection of high viral load in the brainstem after SARS$\mathrm{CoV}$ infection is also indicative of infection spreading to the CNS from the respiratory tract, which is connected primarily by the vagus nerves to the ambiguous and solitary tract nuclei in the brainstem. Involvement of this brain region may also suggest that the cardiorespiratory center contributes to the severe respiratory distress caused by COVID-19.[11] Importantly, the extent of SARS-CoV2 invasion of the CNS, and its role in the respiratory distress and failure needs further investigation. A single study has reported enhanced antibody responses against different forms of coronaviruses in the cerebrospinal fluid of patients with PD compared to other neurological diseases and healthy controls. [12]

\section{NON-MOTOR SYMPTOMS OF PARKINSON' DISEASE}

In recent years, non-motor disorders have attracted increasing interest. $[13,14]$ However, a study showed that people with PD tend to exhibit more non-motor symptoms compared to normal controls (estimated at an average of 8.4 vs. 2.8). These non-motor symptoms, in affected individuals, tend to be more frequent, more bothersome and more severe than the classic triad which is the motor symptoms.[15] Therefore, Behavioural disturbances are frequently observed in PD, including mood and anxiety disorders. With a prevalence of $40 \%$, the depression is the most common psychiatric disturbance reported in PD. [16] As a consequence there are many and serious human, social and health care impacts associated with the onset of depression in PD patients, mainly: anhedonia, desperation, social isolation, self-deprecation, dysphoria, irritability, symptoms of anxiety, which can aggravate into suicidal ideation and death. [17] However, PD related depression remain potentially untreated, despite that it may also result in a poor prognosis and predict a more rapid deterioration of cognitive and motor functions.[18]

\section{SOCIAL COGNITION DYSFUNCTIONS IN PARKINSON'S DISEASE: NEUROANATOMICAL CORRELATES AND CLINICAL IMPLICATIONS}

SOCIAL cognition is defined as a wide range of cognitive capacities elicited by, about, and directed towards other people.[19] In particular, these skills allow humans to both understand themselves end engage in interactions with and understand others, and develop appropriate goal-directed behaviors.[19] Given that social cognition may play a prominent role in clinical care of most psychiatric and neurological illnesses,[20] recent studies addresses the neurobiological processes underlying social interactions and the behavioral correlates of the disruption of these processes. Indeed, growing evidence suggests that neurodegenerative diseases (NDs) such as PDs are associated with social impairments leading to the disruption of interpersonal relationships, thereby eliminating the benefits that social interactions may have for patients with other cognitive impairments. In this chapter, we discuss the basic components of social cognition referring to recent hypotheses derived from network-based approaches, and the clinical manifestations of social cognitive dysfunctions in PDs.

\section{SOCIAL COGNITION, SOCIAL BEHAVIOR, AND SOCIAL FUNCTIONING}

According to the theory of mind (ToM) higher-order functions, such as interpreting other people's mental states, regulating emotions and feelings, and experiencing complex affective behaviors require processing a specific set of stimuli (social stimuli) including facial and/or vocal emotional expressions, body posture.[19, 21, 22] "Social cognition" implies any cognitive processing (e.g., perception, reasoning, memory, attention, motivation, and decision-making) in the context of social domain. It causes "social behavior" that comprises the readily observable interactions between different individuals, while "social 
functioning" consists in the long-term and contextualized ability of an individual to interact with other people.[23]

\section{NETWORK-BASED APPROACH TO SOCIAL COGNITION}

Data from Functional Resonance Imaging (fMRI) studies and neurological and psycho-affective disorders provided insights about the brain structures and networks implicated in social processing and behavior (i.e., ventromedial and dorsomedial prefrontal cortex, temporoparietal junction and superior temporal cortex, insula, and fusiform gyrus).[19, 24] However, we should point out that no social process can be attributed to a single structure but rather to a distributed interconnected.

Thus, evidence collected from resting state fMRI (rsfMRI) studies helped developing a recent network-based approach. $[19,24,25]$ Research showed that the amygdala plays a pivotal role in triggering emotional responses, detecting socially salient stimuli and performing social affiliative behaviors.[22, 26] The neuroanatomy supports the position of the amygdala as a core component of the social network.[22, 27] This network comprises most structures of the social brain (i.e., medial prefrontal cortex (PFC), orbitofrontal cortex (OFC), anterior cingulate cortex, temporoparietal junction, inferior frontal gyrus, and superior temporal sulcus). A second network involved in social cognition is the so-called "mentalizing" network, which includes the right temporoparietal junction as a key region,[28] found to be activated in the case of moral judgements.[29-31] The empathy network, the third circuit implicated in social cognition and behavior, includes the cingulo-insular structures.[32] Finally, the so-called "mirror neuron system", mainly involving the inferior frontal gyrus, the inferior parietal lobule, the fusiform face area, and the superior temporal sulcus, $[32,33]$ is activated during the observation of the actions of others, including emotion recognition. [34, 35]

\section{SOCIAL COGNITION ABNORMALITIES IN PARKINSON'S DISEASE AND IN PARKINSONISM} Several studies revealed deficits in emotion recognition in PD patients with respect to healthy controls.[36-38] However, other studies failed to confirm these deficits.[39, 40] A meta-analytic review,[41] investigated facial and vocal emotion recognition of PD patients, revealed significant and modest alterations of this ability independently from the level of motor symptoms. Moreover, it has been reported that PD patients were more impaired in recognizing negative emotions (anger, disgust, fear, and sadness) than positive ones (happiness, surprise),[42] while other studies suggested that the recognition of negative emotions may be impaired mainly in the early stages of PD and, then, this impairment has been shown to mainly affect the positive ones. [43] Interestingly, impairments of facial emotion recognition in PD patients were found to be independent of depressive symptoms, executive deficits, and clinical aspects (i.e., disease duration and severity).[44-46] Moreover, some studies revealed that emotion recognition abnormalities may occur after subthalamic nucleus stimulation, [47, 48] [49] probably due to alterations of projections to cortical areas, particularly the OFC, which has been already implicated in emotion recognition.[21] However, the study of Albuquerque et al. (2014) did not confirm these findings in advanced PD.[50]

\section{NEUROANATOMICAL BASES OF SOCIAL COGNITION IMPAIRMENT IN PD AND PARKINSONISM}

Concerning the structural neural bases of the deficits in facial emotion recognition, Ibarretxe-Bilbao et al. (2009)[51] revealed an association between these deficits and the degeneration of OFC and amygdala. More recently, Baggio et al. (2012)[52] confirmed that insults in one of these areas may underlie the impairment of facial emotion recognition.

Previous neuroimaging studies, focusing on functional changes associated with the impaired recognition of emotions in PD patients, revealed that the impaired emotional facial recognition network was characterized by a decreased metabolism within the bilateral posterior cingulate gyrus (BA 31), right superior frontal gyrus (BAs 10,9 , and 6), and left superior frontal gyrus (BAs 10 and 11). [53] Furthermore, Wabnegger et al. (2015) found that, when compared to healthy subjects, PD patients showed a stronger activation in somatosensory cortices, which are involved in decoding emotional states by internally generating somatosensory representations that simulate how we feels when displaying a certain facial expression and, therefore, may be substantially involved in emotion recognition.[54]

With regard to parkinsonisms, impaired emotion recognition in PSP patients have been associated with gray matter atrophy in the right inferior frontal gyrus.[55] PSP patients have been proven to exhibit mild but significant focal deficits in social cognition,[56] which is consistent with evidence showing that they may often manifest behavioral and personality changes, hypothesized to occur as a result of a disconnection between subcortical structures and the prefrontal cortex .[57, 58]

\section{PSYCHOLOGICAL OUTCOMES OF SOCIAL DISTANCING AND HEALTH CONTAINMENT: THE GENERAL POPULATION}

The Public health emergencies can affect the health, safety and well-being of individuals (causing, for example, insecurity, confusion, emotional and social isolation).[59] The Covid-19 Pandemic, similar to other public Health emergencies, had alarming consequences for both individual and community health and emotional and behavioural outcomes. The pandemic situation caused by the outbreak of the coronaviruses disease 2019 (Covid-19) is a source of stress and anxiety for many people around the world. In china, more than half participants in a survey have rated moderate-to-severe psychological impact of the outbreak.[60]

In Morocco, the measures taken by the government, social distancing, and partial health containment, have probably contributed to the relative deceleration in the propagation of this pandemic since March $29^{\text {th }}, 2020$. These measures are necessary because limiting social contact of affected people is crucial for the control of COVID-19, as asymptomatic or mildly symptomatic patients can spread the virus. However, these public health measures enhance the probability of adverse psychological consequences in the population. In addition, anxiety reactions are becoming more frequent, as people fear falling ill or seeing their family members falling sick. [61] 
The Social distancing, also called physical distancing, means to keep a safe distance and limit the close contact between people, in order to avoid the spread of the infection. Many instructions have been recommended by the Centers for Disease Control (CDC) in order to fight the coronavirus pandemic, the social distancing measure is one of the best tool we can use to limit the risk of personperson transmission.[62] Moreover, the impact of the social distancing may make the mental health even worse, especially the ones that are psychologically vulnerable. In 2015, after the spread of the Middle East Respiratory Syndrome (MERS), the people that were isolated for two weeks due to their contact with MERS patients, expressed high rates of anxiety and anger, and they suffered mentally even at four-six months after the isolation.[63]

The social relations are integral part of the human wellbeing, and are that are critically involved in the maintenance of health. It has been suggested that satisfaction with social relationships influences mental well-being and depressive symptoms in older adults.[64] Some research has shown that socially isolated people can request visits to the doctor, but not for medical reasons, but to meet their need for interaction and interpersonal stimulation.[65] However, the research consistently documents social isolation is have been linked to the development of morbidity and mortality risk.[66] Cacioppo and Hawkley.(2020) indicated that a sense of isolation or loss of social relationships is associated with declining cognition and mood, along with increased cortisol levels, deterioration in immune function, sleep disturbance, and other social problems.[67] In previous studies, social disconnection has been shown to independently predict the severity of symptoms of depression and anxiety.[68]

In addition, the anxiety can emerge also from the fear of contagion and lack in clarity about the guidelines on social distancing, and is often exacerbated for less reliable media sources that increase confusion and fear. [69]

\section{EFFECTS OF SOCIAL DISTANCING ON PD PATIENTS}

The older people and the ones with serious underlying medical conditions might be at a high risk for severe illness from coronavirus.[70] A previous study reported that the chronic hypertension and other cardiovascular comorbidities were more common in descent patients (from Covid-19) than the recovered ones. [71] To the best of our knowledge, there is no evidence that the patients with movement disorders are at increased risk covid-19. However, the persons with neurological affections, are in general, more susceptible to the risk of the infection.[72] Perhaps the immune response is more disturbed which might be a predisposing factor. In addition, patients with severe infection were more likely to develop neurological manifestations.[73] These particular concerns around the vulnerability of the people with neurological disorders to the infection, creates a stressful situation for this category that might be more worsen either by receiving a diagnostic for covid-19, or/and by being quarantined. Certain populations may be more susceptible than others to the psychosocial effects of pandemics. People with preexisting psychiatric disorders, like Parkinson's patients, are at increased risks of adverse psychosocial consequences.
Parkinson's disease (PD) which is the second most common neurodegenerative affections, being common in the elderly, and associated to pulmonary complications, [74] make the parkinsonian patients more susceptible to develop severe forms of COVID19. On the same line, in a group of patients, PD was associated with a higher risk of dying from pneumonia than controls from the general population.[75]

As the SARS-CoV-2 virus continues to spread across the globe, many countries have taken drastic measures to slow down infection rates. Such drastic policies caused fast changes on people's daily life, requiring them to develop flexible adaptations to the new circumstances. Interestingly, it has been suggested that the cognitive processes underlying behavioral adaptability are dopamine-dependent.[76] Generally, PD is characterized by destruction of dopaminergic neurons in the substantia nigra pars compacta (SNpc), and it has been estimated that the clinical symptoms appear after the depletion of 70-80\% of the striatal dopamine level.[77] Previous studies have shown that PD patients experience cognitive and motor inflexibility, as a result of nigrostriatal dopamine depletion.[78, 79] Thus, the pathophysiology of PD increased the risk of experiencing chronic stress. we argue that increased levels of stress during the COVID-19 pandemic may have several short-term as well as longterm adverse consequences for individuals with PD. Indeed, it has been hypothesized that the dopamine deficiency may lead to increased psychological stress levels and a sense of loss of control in PD.[76] This explains the high incidence of depression disturbance in patients with PD (40 to 50\%).[80] Certainly, the stress level is increasing with the current Covid-19 pandemic, which may make PD patients experience increased levels of chronic stress. It has been revealed previously that the exposure a psychological stress increases the severity of the PD symptoms both in human[81] and animal model.[82] In fact, psychological stress can temporarily worsen various motor symptoms, such as tremor, freezing of gait or dyskinesias,[7, 83, 84] while it has been suggested that it may reduce the efficacy of dopaminergic medication [7]. We should also highlight that increased stress may unmask a latent hypokinetic rigid syndrome, possibly by depleting compensatory mechanisms. $[85,86]$ Moreover, chronic stress exposure along with neurotoxin induced-neurodegeneration, was shown to result not only in behavioral disruption but also in exacerbated degeneration of dopaminergic neurons within the nigrostriatal pathway.[87]

Physical activity (PA) is increasingly advocated to individuals with psychological and movement disorders. It can be seen as a complement to the pharmaceutical treatment in several disease. A single-blind study showed that and exercise program could improve the motor, psychiatric and well-being in patients with Psychogenic movement disorders. [88] In addition, an adapted activity program for patients with PD could be an effective complement therapy to improve the quality of life. [89] Moreover, several studies have discussed the positive effect of the PA on people living with PD and its importance in maximizing the effectiveness of treatments. $[8,90]$

Recent studies have shown that physical exercise may attenuate clinical symptom progression in PD .[91, 92] 
One recent trial compared a home-based physical aerobic exercise intervention with an active control condition, [92] the study reported a between-group longitudinal difference of 4.2 points on the motor scores of the Unified Parkinson's Disease Rating Scale (MDSUPDRS) which was both significant and clinically relevant. Other studies showed that the outcomes of the physical activity depend on the intensity of the exercise; the more intense the activity was, the better were the outcomes.[91] However, it is still unclear which mechanisms control these clinical effects, and in particular whether aerobic exercise can potentially slow down disease progression of $\mathrm{PD}$, or whether it is merely a symptomatic effect via stimulation of compensatory cerebral changes that balance nigrostriatal cell loss. In the present paper, we urge healthcare provider to encourage and motivate their PD patients to practice indoor activities such as mindfulness yoga programs since they were found to reduce significantly anxiety and depression levels in PD patients.[93]

\section{SUMMARY AND FUTURE DIRECTIONS}

Finally, this paper supports the idea that this pandemic would have tremendous long-term outcomes that could persist even after the end of the outbreak. The entire world will suffer heavy losses in the health sector, and more intensively in terms of mental health. Social distancing and health confinement will have a major impact on the psychological state of the overall population worldwide. The psychological disorders (depression and anxiety) in Parkinson's patients are very likely to develop during the pandemic which may exacerbate and make their motor dysfunctions even worse. Being exposed to such chronic stress, an increasing in the incidence of PD patients may be expected after this crisis. These outcomes point up the necessity for the health services to be aware of the mental health of patients with PD during the COVID-19 epidemic. Moreover, the reduction of physical exercises may worsen the movement disorders and also the mental health of the patients. Even though we are living a hard situation, there is always something that can be and needs to be done, for example, clinicians should follow their patients using the teleconsultation techniques, and give the priority to their psychological state more than ever before. Moreover, the PD patients must be encouraged to follow the instructions recommended by the WHO, but also they need to be motivated to respect a home-based activity program. The patient's family and friends are expected to keep their beloved ones away from the COVID 19 news, since they are just a source of anxiety or distress for them, and to avoid as much as possible sharing with them any rumours or misinformation. These measures may help to minimize the following psychological disorders and may contribute in the increasing of the treatment efficacy. After containment, this literature review must be complemented with research about the psychological impact of this pandemic on parkinsonian patients and the evolution of the motor symptoms.

\section{AUTHORS' CONTRIBUTIONS}

The participation of each author corresponds to the criteria of authorship and contributorship emphasized in the Recommendations for the Conduct, Reporting, Editing, and Publication of Scholarly work in Medical Journals of the International Committee of Medical Journal Editors. Indeed, all the authors have actively participated in the redaction, the revision of the manuscript, and provided approval for this final revised version.

\section{COMPETING INTERESTS}

The authors declare no competing interests with this case.

\section{FUNDING SOURCES}

This work was funded by the Laboratory of Cell Biology and Molecular Genetics, Department of Biology, Faculty of Sciences, Ibn Zohr University, Agadir, Morocco. 


\section{REFERENCES}

[1] Monti, C., et al., Systems biology analysis of the proteomic alterations induced by MPP+, a Parkinson's disease-related mitochondrial toxin. J Frontiers in cellular neuroscience, 2015. 9: p. 14.

[2] Gallagher, D.A., A.J. Lees, and A. Schrag, What are the most important nonmotor symptoms in patients with Parkinson's disease and are we missing them? J Movement Disorders, 2010. 25(15): p. 2493-2500.

[3] Schrag, A., Psychiatric aspects of Parkinson's disease. J Journal of neurology, 2004. 251(7): p. 795-804.

[4] Papa, S.M., et al., Impact of the COVID-19 pandemic on Parkinson's disease and movement disorders. J Mov Disord, 2020. 6

[5] COVID-19. CORONAVIRUS PANDEMIC. 2020; Available from: Available from : https://www.worldometers.info/coronavirus/ consulted on 15 Mai 2020.

[6] Khan, S., et al., Impact of coronavirus outbreak on psychological health. J Journal of Global Health, 2020. 10(1)

[7] Zach, H., et al., Cognitive stress reduces the effect of levodopa on Parkinson's resting tremor. J CNS neuroscience therapeutics, 2017. 23(3): p. 209-215.

[8] Bhalsing, K.S., M.M. Abbas, and L.C. Tan, Role of physical activity in Parkinson's disease. J Annals of Indian Academy of Neurology, 2018. 21(4): p. 242.

[9] Bravo, D., et al., Effect of the IL28B Rs12979860 C/T polymorphism on the incidence and features of active cytomegalovirus infection in allogeneic stem cell transplant patients. J Journal of medical virology, 2014. 86(5): p. 838-844.

[10] Li, K., et al., Middle East respiratory syndrome coronavirus causes multiple organ damage and lethal disease in mice transgenic for human dipeptidyl peptidase 4. J The Journal of infectious diseases, 2016. 213(5): p. 712-722.

[11] Matsuda, K., et al., The vagus nerve is one route of transneural invasion for intranasally inoculated influenza a virus in mice. J Veterinary pathology, 2004. 41(2): p. 101107.

[12] Fazzini, E., J. Fleming, and S. Fahn, Cerebrospinal fluid antibodies to coronavirus in patients with Parkinson's disease. J Movement disorders: official journal of the Movement Disorder Society, 1992. 7(2): p. 153-158.

[13] Tibar, H., et al., Non-motor symptoms of Parkinson's disease and their impact on quality of life in a cohort of Moroccan patients. J Frontiers in neurology, 2018.9:p. 170.

[14] Bugalho, P., et al., Non-Motor symptoms in Portuguese Parkinson's Disease patients: correlation and impact on Quality of Life and Activities of Daily Living. J Scientific reports, 2016. 6: p. 32267.

[15] Pfeiffer, R.F., Non-motor symptoms in Parkinson's disease. J Parkinsonism related disorders, 2016. 22: p. S119-S122.

[16] Oh, S.-i., et al., Neuroanatomical correlates of depressive symptoms in newly diagnosed Parkinson's disease patients. J Neurology Asia, 2018. 23(3).

[17] Dumitru, M. and A. Papari. Suicide attempts, suicidal and death ideation in Parkinson's disease. in MOVEMENT DISORDERS. 2018. WILEY 111 RIVER ST, HOBOKEN 07030-5774, NJ USA

[18] Burn, D.J., Depression in Parkinson's disease. J European Journal of Neurology, 2002. 9: p. 44-54.

[19] 19. Adolphs, R., The social brain: neural basis of social knowledge. J Annual review of psychology, 2009. 60: p. 693-716

[20] Cacioppo, S., J.P. Capitanio, and J.T. Cacioppo, Toward a neurology of loneliness. J Psychological bulletin, 2014. 140(6): p. 1464

[21] Adolphs, R., S. Baron-Cohen, and D. Tranel, Impaired recognition of social emotions following amygdala damage. J Journal of cognitive neuroscience, 2002. 14(8): p. $1264-1274$.

[22] Adolphs, R., What does the amygdala contribute to social cognition? J Annals of the New York Academy of Sciences, 2010. 1191(1): p. 42.

[23] Henry, J.D., et al., Clinical assessment of social cognitive function in neurological disorders. J Nature Reviews Neurology, 2016. 12(1): p. 28.
[24] Brothers, L.J.F.i.s.n., The social brain: a project for integrating primate behavior and neurophysiology in a new domain. 2002. 367: p. 385

[25] Haxby, J.V., E.A. Hoffman, and M.I. Gobbini, The distributed human neural system for face perception. J Trends in cognitive sciences, 2000. 4(6): p. 223-233.

[26] Young, A.W., et al., Face processing impairments after amygdalotomy. J Brain, 1995. 118(1): p. 15-24.

[27] 27. Pessoa, L., On the relationship between emotion and cognition. J Nature reviews neuroscience, 2008. 9(2): p. 148-158.

[28] Hill, C.A., et al., A causal account of the brain network computations underlying strategic social behavior. J Nature neuroscience, 2017. 20(8): p. 1142

[29] Young, L. and R. Saxe, An FMRI investigation of spontaneous mental state inference for moral judgment. J Journal of cognitive neuroscience, 2009. 21(7): p. 13961405 .

[30] Saxe, R., Why and how to study Theory of Mind with fMRI. J Brain research, 2006. 1079(1): p. 57-65.

[31] Dodell-Feder, D., et al., fMRI item analysis in a theory of mind task. J Neuroimage, 2011. 55(2): p. 705-712.

[32] Iacoboni, M., et al., Cortical mechanisms of human imitation. J science, 1999. 286(5449): p. 2526-2528.

[33] Decety, J., et al., A PET exploration of the neural mechanisms involved in reciprocal imitation. J Neuroimage, 2002. 15(1): p. 265-272.

[34] Enticott, P.G., et al., Mirror neuron activation is associated with facial emotion processing. J Neuropsychologia, 2008. 46(11): p. 2851-2854.

[35] Keuken, M., et al., The role of the left inferior frontal gyrus in social perception: an rTMS study. J Brain research, 2011. 1383: p. 196-205.

[36] Beatty, W.W., et al., Affective judgments by patients with Parkinson's disease or chronic progressive multiple sclerosis. J Bulletin of the Psychonomic Society, 1989. 27(4): p. 361-364.

[37] Ariatti, A., F. Benuzzi, and P. Nichelli, Recognition of emotions from visual and prosodic cues in Parkinson's disease. J Neurological Sciences, 2008. 29(4): p. 219.

[38] De Risi, M., et al., Facial emotion decoding in patients with Parkinson's disease. J International Journal of Neuroscience, 2018. 128(1): p. 71-78.

[39] Adolphs, R., R. Schul, and D. Tranel, Intact recognition of facial emotion in Parkinson's disease. J Neuropsychology, 1998. 12(2): p. 253.

[40] Ille, R., et al., Intact emotion recognition and experience but dysfunctional emotion regulation in idiopathic Parkinson's disease. J Journal of the neurological sciences, 2016. 361: p. 72-78.

[41] Gray, H.M. and L. Tickle-Degnen, A meta-analysis of performance on emotion recognition tasks in Parkinson's disease. J Neuropsychology, 2010. 24(2): p. 176.

[42] Sedda, A., et al., Identification and intensity of disgust: distinguishing visual, linguistic and facial expressions processing in Parkinson disease. $\mathrm{J}$ Behavioural brain research, 2017. 330: p. 30-36.

[43] Lin, C.-Y., et al., Degraded impairment of emotion recognition in Parkinson's disease extends from negative to positive emotions. J Behavioural neurology, 2016. 2016.

[44] Saenz, A., et al., Recognition of facial and musical emotions in $\mathrm{P}$ arkinson's disease. J European journal of neurology, 2013. 20(3): p. 571-577.

[45] Enrici, I., et al., Emotion processing in Parkinson's disease: a three-level study on recognition, representation, and regulation. J PLoS One, 2015. 10(6).

[46] Pietschnig, J., et al., Facial emotion recognition and its relationship to cognition and depressive symptoms in patients with Parkinson's disease. J International psychogeriatrics, 2016. 28(7): p. 1165-1179.

[47] Drapier, D., et al., Emotion recognition impairment and apathy after subthalamic nucleus stimulation in Parkinson's disease have separate neural substrates. J Neuropsychologia, 2008. 46(11): p. 2796-2801.

[48] Biseul, I., et al., Fear recognition is impaired by subthalamic nucleus stimulation in Parkinson's disease. J Neuropsychologia, 2005. 43(7): p. 1054-1059. 
[49] Péron, J., et al., Subthalamic nucleus stimulation affects fear and sadness recognition in Parkinson's disease. 2010. 24(1): p. 1.

[50] Albuquerque, L., et al., STN-DBS does not change emotion recognition in Parkinson's disease. J Parkinsonism related disorders, 2014. 20(5): p. 564.

[51] Ibarretxe-Bilbao, N., et al., Neuroanatomical correlates of impaired decision-making and facial emotion recognition in early Parkinson's disease. J European Journal of Neuroscience, 2009. 30(6): p. 1162-1171.

[52] Baggio, H., et al., Structural correlates of facial emotion recognition deficits in Parkinson's disease patients. J Neuropsychologia, 2012. 50(8): p. 2121-2128.

[53] Robert, G., et al., Apathy and impaired emotional facial recognition networks overlap in Parkinson's disease: a PET study with conjunction analyses. J Neurol Neurosurg Psychiatry, 2014. 85(10): p. 1153-1158.

[54] Wabnegger, A., et al., Facial emotion recognition in Parkinson's disease: an fMRI investigation. J PLoS One, 2015. 10(8).

[55] 55. Ghosh, B.C., et al., Social cognitive deficits and their neural correlates in progressive supranuclear palsy. J Brain, 2012. 135(7): p. 2089-2102.

[56] Shany-Ur, T., et al., Comprehension of insincere communication in neurodegenerative disease: lies, sarcasm, and theory of mind. J Cortex, 2012. 48(10): p. 1329-1341.

[57] Grafman, J., et al., Frontal lobe function in progressive supranuclear palsy. J Archives of Neurology, 1990. 47(5): p. 553-558.

[58] Kaat, L.D., et al., Frontal presentation in progressive supranuclear palsy. J Neurology Asia, 2007. 69(8): p. 723729.

[59] Pfefferbaum, B. and C.S. North, Mental health and the Covid-19 pandemic. J New England Journal of Medicine, 2020.

[60] Wang, C., et al., Immediate psychological responses and associated factors during the initial stage of the 2019 coronavirus disease (COVID-19) epidemic among the general population in China. $\mathrm{J}$ International journal of environmental research, 2020. 17(5): p. 1729.

[61] Index, in Aging, Nutrition and Taste, J.B. Marcus, Editor. 2019, Academic Press. p. 495-513.

[62] Fong, M.W., et al., Nonpharmaceutical measures for pandemic influenza in nonhealthcare settings-social distancing measures. 2020

[63] Jeong, H., et al., Mental health status of people isolated due to Middle East Respiratory Syndrome. J Epidemiology, 2016. 38.

[64] Lund, R., C.J. Nilsson, and K. Avlund, Can the higher risk of disability onset among older people who live alone be alleviated by strong social relations? A longitudinal study of non-disabled men and women. J Age Ageing, 2010. 39(3): p. 319-326

[65] Gerst-Emerson, K. and J. Jayawardhana, Loneliness as a public health issue: the impact of loneliness on health care utilization among older adults. J American journal of public health, 2015. 105(5): p. 1013-1019.

[66] Steptoe, A., et al., Social isolation, loneliness, and all-cause mortality in older men and women. J Proceedings of the National Academy of Sciences, 2013.110(15): p.5797-801.

[67] Cacioppo, J.T. and L.C. Hawkley, Perceived social isolation and cognition. $\mathrm{J}$ Trends in cognitive sciences, 2009. 13(10): p. 447-454.

[68] Newman, M.G. and N.H. Zainal, The value of maintaining social connections for mental health in older people. J The Lancet Public Health, 2020. 5(1): p. e12-e13.

[69] Venkatesh, A. and S. Edirappuli, Social distancing in covid-19: what are the mental health implications? J Bmj, 2020. 369.

[70] Guan, W.-j., et al., Clinical characteristics of coronavirus disease 2019 in China. J New England journal of medicine, 2020. 382(18): p. 1708-1720.

[71] Chen, T., et al., Clinical characteristics of 113 deceased patients with coronavirus disease 2019: retrospective study. J Bmj, 2020. 368.
[72] Stoessl, A.J., K.P. Bhatia, and M. Merello, Movement Disorders in the World of COVID-19. J Movement Disorders Clinical Practice, 2020.

[73] Mao, L., et al., Neurologic manifestations of hospitalized patients with coronavirus disease 2019 in Wuhan, China. J JAMA neurology, 2020

[74] Woodford, H. and R. Walker, Emergency hospital admissions in idiopathic Parkinson's disease. J Movement disorders: official journal of the Movement Disorder Society, 2005. 20(9): p. 1104-1108.

[75] Beyer, M., et al., Causes of death in a community-based study of Parkinson's disease. J Acta Neurologica Scandinavica, 2001. 103(1): p. 7-11.

[76] Douma, E.H. and E.R. de Kloet, Stress-induced plasticity and functioning of ventral tegmental dopamine neurons. $\mathrm{J}$ Neuroscience Biobehavioral Reviews, 2020. 108: p. 48-77.

[77] Postuma, R., et al., How does parkinsonism start? Prodromal parkinsonism motor changes in idiopathic REM sleep behaviour disorder. J Brain, 2012. 135(6): p. 18601870 .

[78] Helmich, R.C., et al., Increased dependence of action selection on recent motor history in Parkinson's disease. J Journal of neuroscience, 2009. 29(19): p. 6105-6113.

[79] Robbins, T.W. and R. Cools, Cognitive deficits in Parkinson's disease: a cognitive neuroscience perspective. J Movement Disorders, 2014. 29(5): p. 597-607.

[80] Marsh, L., Depression and Parkinson's disease: current knowledge. J Current neurology neuroscience reports, 2013. 13(12): p. 409

[81] Macht, M., R. Schwarz, and H. Ellgring, Patterns of psychological problems in Parkinson's disease. J Acta Neurologica Scandinavica, 2005. 111(2): p. 95-101.

[82] Janakiraman, U., et al., Influences of chronic mild stress exposure on motor, non-motor impairments and neurochemical variables in specific brain areas of $\mathrm{MPTP} /$ probenecid induced neurotoxicity in mice. $\mathrm{J}$ PloS one, 2016. 11(1).

[83] Macht, M., et al., Predictors of freezing in Parkinson's disease: a survey of 6,620 patients. J Movement Disorders, 2007. 22(7): p. 953-956

[84]Zach, H., et al., The patient's perspective: The effect of levodopa on Parkinson symptoms. J Parkinsonism related disorders, 2017. 35: p. 48-54.

[85] Snyder, A.M., E.M. Stricker, and M.J. Zigmond, Stressinduced neurological impairments in an animal model of parkinsonism. J Annals of neurology, 1985.18(5):p.544-51.

[86] Djamshidian, A. and A.J. Lees, Can stress trigger Parkinson's disease? J Neurol Neurosurg Psychiatry, 2014. 85(8): p. 878-881.

[87] Hemmerle, A., et al., Stress exacerbates experimental Parkinson's disease. J Molecular psychiatry, 2014. 19(6): p. 638-640.

[88] Dallocchio, C., et al., The effects of physical activity on psychogenic movement disorders. J Movement disorders, 2010. 25(4): p. 421-425.

[89] Cugusi, L., et al., Effects of an adapted physical activity program on motor and non-motor functions and quality of life in patients with Parkinson's disease. J NeuroRehabilitation, 2014. 35(4): p. 789-794.

[90] Goodwin, V.A., et al., The effectiveness of exercise interventions for people with Parkinson's disease: A systematic review and meta-analysis. J Movement disorders, 2008. 23(5): p. 631-640.

[91] Schenkman, M., et al., Effect of high-intensity treadmill exercise on motor symptoms in patients with de novo Parkinson disease: a phase 2 randomized clinical trial. J JAMA neurology, 2018. 75(2): p. 219-226.

[92] van der Kolk, N.M., et al., Effectiveness of home-based and remotely supervised aerobic exercise in Parkinson's disease: a double-blind, randomised controlled trial. J The Lancet Neurology, 2019. 18(11): p. 998-1008.

[93] Kwok, J.Y., et al., Effects of mindfulness yoga vs stretching and resistance training exercises on anxiety and depression for people with Parkinson disease: A randomized clinical trial. J JAMA neurology, 2019. 76(7): p. $755-763$. 